\title{
Variation in Nutrient Intakes and Required Number of Days for Assessing Usual Nutrient Intake among Different Populations
}

\author{
Ock K. Chun* and Catherine G. Davis
}

Department of Nutritional Sciences, University of Connecticut, Storrs, CT 06269

\begin{abstract}
Estimating the usual intake of a population is essential in the process of establishing the protective effects of nutrients against the development of certain diseases and assessing nutrient intake adequacy. A long term assessment of daily intakes is required to assess usual intakes; however, due to the cost and burden of this requirement, most studies employ shorter term assessments. Many limitations exist with dietary assessment tools which can alter the results and conclusions of intake data. This article was aimed to review on the variation of nutrient intakes and required number of days to assess usual nutrient intake status of diverse population groups based on previous publications.
\end{abstract}

Keywords: Usual nutrient intake; Misreporting; Within-person variation; Between-person variation; Antioxidants

\section{Introduction}

Estimating the usual intake of a population is essential in the process of establishing the protective effects of nutrients against the development of certain diseases and assessing nutrient intake adequacy [1]. Common assessment tools used in epidemiological studies include diet histories, dietary recalls (DR), food frequency questionnaires (FFQ), and food records (FR) [2]. A long term assessment of daily intakes is required to assess usual intakes; however, due to the cost and burden of this requirement, most studies employ shorter term assessments. Many limitations exist with dietary assessment tools which can alter the results and conclusions of intake data [3]. Examples of important limitations include misreporting nutrient consumption [4] and large within- and between-variation in daily intakes of participants [5] which can weaken the relationship between dietary intake and disease risk factors. Under-reporting of nutrient intake is a significant problem in nutritional epidemiological studies and may increase the estimation of inadequate nutrient consumption as well as affect the interpretation of nutrient distributions and its applications to a population group [6]. Daily intakes can vary greatly from day-to-day often as a result of seasons or cultural or environmental factors [7]. This within-person variation, as well as the variation between individuals, must be estimated to understand the relationship of diet and health status. Statistical methods have been developed to control for these factors to produce accurate estimates of usual intake from shorter recording periods $[1,8-10]$; however, increasing the number of days of dietary assessment greatly decreases the bias associated with this source of variation [5]. In addition, the number of days of diet record necessary to accurately estimate true intake for each nutrient should be carefully considered when designing a study for a specific population group $[11,12]$.

There are few studies that evaluate research methodology for assessing variation and intake of nutrients and non-nutrient antioxidants in the U.S. diet. Therefore this article was aimed to review on the variation of nutrient intakes and required number of days to assess usual nutrient intake status of diverse population groups based on previous publications.

\section{Estimation of Usual Nutrient Intake among Populations}

Estimation of usual or habitual nutrient intake of a population is a vital process in nutritional research. The truest representation of intake for an individual collectively defines usual intake for a population [3]. Usual dietary intakes are required in order to define nutrient intake adequacy, contribute to the baseline data required for dietary guidelines for specific age groups, provide a basis for nutritional interventions, and establish the relationship between diet and health status or risk of disease [2]. Considerable effort has been devoted to analyzing the effects of this latter point, specifically in relation to cancers and cardiovascular disease (CVD). Unfortunately, few studies have defined accurate methods of determining habitual dietary intakes $[5,9,10,13,14]$. Common assessment tools used in epidemiological studies include diet histories, DR, FFQ, and FR [15]. Long term dietary assessments are required to provide an accurate representation of dietary intakes and patterns. FR are often employed to assess usual intake, however, they are rarely used for weeks or months at a time due to participant responsibility and associated errors due to fatigue [3]. Therefore, many studies report nutrient intakes using shorter record periods, which may provide less reliable data [16]. Despite the advances in technology to collect nutrient data from these assessment tools, limitations exist. Major limitations include misreporting of nutrient consumption and variation of intakes from day-to-day and between-individuals, which creates inconsistent results and a gap in the relationship between diet and disease $[17,18]$.

\section{Misreporting of nutrient intakes}

Misreporting by all population groups is a serious issue in nutrition and health related research. It can compromise the accuracy, validity, and application of data reporting nutrient intake. Collection of long term dietary intakes is preferred, but can also increase the likelihood of misreporting intake due to the burden of recording daily intake. The first day of any diet recording period is considered to be the most accurate $[9,19]$. The term misreporting encompasses under-reporting and over-reporting nutrient intake [4]. Under-reporting or low energy reporting can be the result of under estimating food intake, elimination of certain foods or amounts, or under-eating due to dietary restrictions or dieting. Underestimating food intake may be the result

*Corresponding author: Ock K. Chun, PhD, MPH, Assistant Professor Department of Nutritional Sciences, University of Connecticut, 3624 Horsebarn, Road Extension Unit 4017 Storrs, Connecticut 06269-4017, Tel: (860) 4866275; Fax (860) 486-3674; E-mail: ock.chun@uconn.edu

Received June 20, 2012; Accepted July 17, 2012; Published July 19, 2012

Citation: Chun OK, Davis CG (2012) Variation in Nutrient Intakes and Required Number of Days for Assessing Usual Nutrient Intake among Different Populations. J Nutr Disorders Ther 2:118. doi:10.4172/2161-0509.1000118

Copyright: ( 2012 Chun OK, et al. This is an open-access article distributed under the terms of the Creative Commons Attribution License, which permits unrestricted use, distribution, and reproduction in any medium, provided the original author and source are credited. 
of misunderstanding or error in dietary instruction on portion sizes for the participant or lack of cooperation in consistently measuring food intake. In addition, elimination of certain foods or amounts can be the result of the study participant intentionally or unintentionally selecting foods due to the bias of the study towards analysis of certain nutrients [20]. Regardless of classification of misreporting, the inclusion of individuals who provide inaccurate nutrient intakes can alter the results and conclusions significantly. A common method for identifying such individuals is the Goldberg's cut off equation [21]. This equation requires the average energy intake, average basal metabolic rate, and daily physical activity of the population to generate critical values for energy intake applied to the average energy intake of each individual participant. The population critical values are represented by energy intake: BMR estimated ratio (EI $\left.\mathrm{rep}_{\mathrm{rep}} \mathrm{BMR}\right)$.

A review by Black provides a guide for the use of the Goldberg cut off in nutritional assessment research [4]. In the review, Black emphasizes the importance of selecting a physical activity level (PAL) for each population dependent on reported daily physical activity and classifications provided by the World Health Organization (WHO) [22]. For the average student population group, a 1.6-1.7 PAL is suggested for determining energy requirements [22]. In data reported by Black [4], a PAL of 1.7 was used for young adults aged 18-29 y who were predominately non-Hispanic White and participated in moderate leisure activities. However, a high PAL value can inaccurately identify individuals as low energy reporters especially in a study with a small sample size [4]. In order to increase the sensitivity and specificity of the Goldberg cut off in a study with a population size $(n)<100$, the number of days of dietary intake assessment should be increased [23]. However with larger sample sizes, fewer numbers of dietary assessments may be used. Results from the National Health and Nutrition Examination Survey 1988-1994 (NHANES III) for misreporting using the Goldberg cut off include a critical value of 0.9 to 1.54 with a mean $\mathrm{EI}_{\text {rep }}: \mathrm{BMR}$ of 1.36 for all adults. In addition, $18 \%$ of males and $28 \%$ of females were classified as under-reporters of energy intake [6].

After the identification of misreporters in a population group, causality should be determined before the decision to include or exclude individuals from the results. In a recent review, Poslunsna et al. summarized the main causes of errors in 24-hr DR and FR most frequently reported in 38 nutritional studies [24]. Results indicated that the major determinants for misreporting included body mass index (BMI), age and sex, socioeconomic status and education, health related activities, psychological factors, and eating habits. While misreporting includes both underreporting and overreporting of nutrient intake, overreporting was identified less frequently in these studies. The most consistent factor reported in the review was that as BMI increased, a larger percentage of the population was classified as misreporters, specifically among females. In addition, more females than males tended to misreport their nutrient intake [24]. Similar gender results were found in a study with 53 non obese, weight stable adults. They reported $49 \%$ of the females and $14 \%$ of the males were identified as under-reporters from a 7-day DR [19].

It remains unknown whether males tend to underreport less than women do or if their higher energy requirements allow them to rarely fall below the cutoff limits when applied to an entire study population [23]; however, Asbeck et al. reported that the higher percentage of female underreporters was due to restrained eating practices evidenced by scores from an eating practice survey in a normal weight population [19]. Leibman et al. conducted a study with 324 college students analyzing the relationship between dieting practices, gender, and psychological variables such as self image and body perceptions [25].
The results showed that $38 \%$ of females and $13 \%$ of males had dieted to lose weight within the past year and more females reported patterns of disordered eating, such as fat avoidance or replacement, and body dissatisfaction [25]. Body weight dissatisfaction, frequent dieting, and societal pressures seem to be an area of concern in young adult and adult female populations; therefore the validity of dietary assessments from these population groups should be analyzed before average intake results are reported [26].

\section{Within- and between-person variation of nutrient intake}

Day-to-day variability in nutrient intakes can significantly alter the statistical outcomes and interpretations of dietary assessment data. This fluctuation is defined as within-person variation and can be attributed to environmental and cultural factors [27]. Micronutrients have a higher concentration in specific foods and tend to have greater variation due to seasonal variation or the wide array of food choices available in many developed countries when compared to macronutrients which remain more stable in the diet. However, seasonal variation has a greater impact in developing countries where all foods are not as easily accessible [27]. Day of the week sampled by a dietary assessment tool is another source of within-person variation. Energy and protein consumption are typically larger on the weekends compared to the weekdays and should be considered when using 24-hr DR [9]. Withinperson variation can be estimated and must be adjusted for statistically due to its high correlation to the mean of the sample day. This is crucial in the interpretation when the study design only includes a small number of days of dietary intake [5]. However, increasing the number of days of diet recorded can decrease the within-person variation significantly [3]. Another important consideration is a large variation between individuals of a population because it may misconstrue the relationship between nutrient consumption and disease risks $[4,28]$. Between-person variation can be reduced by accounting for certain sociodemographic and lifestyle factors specific to the group of study $[12,27,29]$. The ratio of the within to between variation can be used to further describe the effect of the within-person variation [1]. The greater the variance ratio, the greater the within-person variation in daily intakes.

Several methods have been developed to assess usual dietary intake among populations [1,8-10]; however all methods require estimation of within- and between-person variation. Therefore, these values must be calculated from multiple numbers of diet records or values can be borrowed from an appropriate subset population [1]. Chang et al. analyzed the within- and between-person variation among Taiwan college students who completed a total of three 5-day DR [1]. They found that males had larger within to between ratios for fat, protein, polyunsaturated fatty acid, vitamin A, thiamin, and riboflavin than the females which they attributed to the irregular eating patterns and possible binging of male college students. Females had larger withinperson variation in the intakes of carbohydrates which could be a result from the common practice of dieting or meal skipping in this population group [1]. In another study, Jahns et al. analyzed the effects of gender as well as age and culture on the estimation of within- and betweenperson variation in U.S. and Russian older children and adolescents [29]. Results were reported from nonconsecutive 24-hr DR from the Russia Longitudinal Monitoring Survey (RLMS) and the Continuing Survey of Food Intake by Individuals (CSFII). They analyzed energy intake and 10 additional macro- and micro-nutrients: protein, carbohydrate, fat, calcium, iron, magnesium, thiamin, riboflavin, niacin and the antioxidant nutrient as vitamin C. Among the U.S. population, they found that the girls had higher within-person variation than the boys for all nutrients excluding carbohydrates and the girls had higher 
between-person variation as well. Results pertaining to the differences in age groups reported that the older Russian girls had higher withinperson variation for all nutrients except riboflavin, niacin, and vitamin $\mathrm{C}$ as well as higher between-person variation for all nutrients except magnesium and thiamin than the younger girls. No observable patterns were found among the U.S. age groups for within-person variation but the between-person variation was higher for the older girls for 9 out of the 11 nutrients including vitamin C [29].

In U.S. men and women, Neuhaus et al. analyzed the ratios of within-person variation to between-person variation in different age groups for energy, 3 macronutrients, and 9 micronutrients including vitamin C [30]. They found that as age increased, the variance ratio decreased meaning the within-person variation was greater than the between-person variation. These results were significant among men for most nutrients, however, a decreasing trend was not as apparent for women [30]. Overall, the results seem to indicate that younger adult populations may have larger day-to-day variability in nutrient intake, which has important implications with estimating usual nutrient intakes of a population. While these studies do include within- and between-person variation among adolescents and young adults, there is a gap in the literature pertaining to antioxidant intakes among this age group in the U.S.

Due to the numerous errors associated with dietary intake assessment, it is important to consider analysis through biomarkers as a complimentary methodology. For example, a review by Hudson et al. provided evidence to support the use of tissue and blood fatty acids as biomarkers for intake due to its correlation of precisely controlled nutrient intake [31]. In addition, multiple studies have identified misreporting by verifying intake using doubly labeled water to measure total energy expenditure (TEE) and $24 \mathrm{hr}$ urinary nitrogen to measure protein intake. However, these methodologies are time consuming for the researchers and participants as well as expensive, specifically in reference to the doubly labeled water technique. The measurement of antioxidant intake has also been assessed through biomarkers. For example, carotenoids can be measured in the plasma, serum, or the skin through the use of high performance liquid chromatography (HPLC) [32]. In addition, vitamins $\mathrm{C}$ and $\mathrm{E}$ can be measured using HPLC. The lipid soluble antioxidants, carotenoids and vitamin $\mathrm{E}$ can be measured in adipose tissue; however, due to the procedure involved, participation from eligible subjects may be a challenge [32]. There are limitations associated with the methodologies utilized in measuring biomarkers, which include lack of reproducibility, specificity, variation within- and between- individuals, and lack of detection. Despite the limitations associated with any method of assessing nutrient intake, there is promising research that nutrient intake data used in conjunction with biomarkers can produce valid and reliable results.

\section{The Number of Days Required to Accurately Assess Nutrient Intake}

It is important, when developing a study design, to know how many days of dietary assessment are required to produce accurate and reliable intake results for a population group [11]. To assess usual nutrient intake levels among a population, within- and betweenperson variation should be estimated and included in a calculation to determine sufficient number of diet records necessary to produce accurate results $[11,12]$.

The calculation of days (D) of nutrient intake includes the ratio of within-person variation $\left(\mathrm{Sb}_{\mathrm{w}}\right)$ to between-person variation $\left(\mathrm{Sb}_{\mathrm{b}}\right)$ [33]. The variability in daily nutrient intake among adults has been shown to be greater than the variability between individuals in a study population [11], and the smaller the ratio, the fewer number of days is required to estimate the nutrient intake within a specified level of accuracy $(r)$ between true intake and observed intake [11]. Nelson et al. analyzed data from 18 studies that reported mean nutrient intake, values for within- and between person variation, and the number of days required to estimate true intake within a given accuracy. They included studies with populations aging from infancy to older adults and reported a total of 29 nutrients including energy. Values presented for $\mathrm{D}$ were based on $\mathrm{r}=0.9$. Most nutrients required more than 7 days of DR to estimate true intake in all age and gender groups. Among the adult populations, energy, protein, carbohydrate, and fat required 4-8 days depending on gender. Females required more days than males for all macronutrients. Vitamin A and carotene were reported to require three weeks or more to estimate true intakes with adult females requiring over a month to estimate carotene. Results pertaining to vitamin $C$ included 12 days for males and 7 days for females while vitamin $\mathrm{E}$ required 8 days for males and 16 days for females. In general, this study found that the population group that required the most days to estimate true intake was 5-17 y with adults requiring an intermediate amount [11].

Mennen et al. reported analysis of the number of 24-hr DR required among French adults participating in the Supplémentation en Vitamines et Minéraux Antioxydants (SU.VI.MAX) Study which investigated the effects of antioxidant supplementation on cancer and heart disease [34]. Participants included in the additional analysis completed six 24-hr DRs over a year and the study was separated into two phases consisting of 2 years each. Nutrients included energy and macronutrients with vitamin $\mathrm{C}$, vitamin $\mathrm{E}$, and $\beta$-carotene as the antioxidant micronutrients. Results from the first phase included the highest variance ratio for $\beta$-carotene and the lowest for carbohydrate. Carbohydrate required 5 days of DR while $\beta$-carotene required 16 recalls. For protein, total fat, and vitamin $C$, results showed $8 \mathrm{DR}$ would be needed while vitamin E required 10 recalls for this French adult population. In general, the women required the same or more DR to estimate true intake for the macro- and micro-nutrients included [34].

A study was conducted in preschool age children reporting the variation in macronutrients and 11 micronutrient intakes stratified by age groups and gender [12]. Huybrechts et al. concluded that as the age of the children increased, the larger the variance ratios became and more days of DR were required for all nutrients. A 7-day DR would be sufficient to estimate energy and macronutrients when analyzing gender; however, results from the age groups indicate than more than 7 days would be required for the older children. Vitamin C could be estimated in 5 days among all age groups and genders [12]. In an older adult population in Korea, the number of days to estimate energy, protein, fat, and carbohydrates among males was over 2 weeks; however, vitamin $\mathrm{C}$ required 54 days to estimate true intake [27]. Females required 8-23 days to estimate their macronutrient intake while vitamin $C$ required 16 days. Oh et al. concluded these results were attributed to the large within-person variability and low betweenperson variability in this population group [27]. Due to the population demographic, many of these studies can only serve as implications for study design. There is limited data on nutrient variability and number of days needed to assess nutrient intake, including antioxidants, among populations of interest.

\section{Conclusion}

Estimation of usual nutrient intake from dietary sources among a target population is a vital part in assessing dietary quality and the 
risk of developing diseases. In order to do so, nutrient intake data must be validated by identifying misreporters and determining the variation among this population. It should be noted that an important application of these methods of validation and estimation of variance components of nutrient intake among a population of study is the evaluation of nutrient intake adequacy according to the Dietary Reference Intakes (DRIs) and the Dietary Guidelines for Americans. While research is often devoted to assessing diet quality and disease risk factors in adults, there is limited data in comparison focusing on this significant population group. With the steady increase of obesity and other diet related illnesses, it is critical that research professionals report accurate nutrient intake in order to link diet and disease risk.

\section{References}

1. Chang HY, Suchindran CM, Pan WH (2001) Using the overdispersed exponential family to estimate the distribution of usual daily intakes of people aged between 18 and 28 in Taiwan. Stat Med 20: 2337-2350.

2. Conway JM, Ingwersen LA,Vinyard BT, Moshfegh AJ (2003) Effectiveness of the US Department of Agriculture 5-step multiple-pass method in assessing food intake in obese and nonobese women. Am J Clin Nutr 77:1171-1178.

3. Willett W (1998) Nutritional Epidemiology $2^{\text {nd }}$ edn. Oxford: OUP

4. Black AE (2000) Critical evaluation of energy intake using the Goldberg cut-off for energy intake:basal metabolic rate. A practical guide to its calculation, use, and limitations. Int J Obes Relat Metab Disord 24: 1119-1130.

5. Carriquiry AL (2003) Estimation of usual intake distributions of nutrients and foods. J Nutr 133: 601S-608S.

6. Briefel RR, Sempos CT, McDowell MA, Chien S, Alaimo K (1997) Dietary methods research in the third national health and nutrition examination survey: Underreporting of energy intake. Am J Clin Nutr 65: S1203-S1209.

7. Jahns L, Arab L, Carriquiry A, Popkin BM (2005) The use of external withinperson variance estimates to adjust nutrient intake distributions over time and across populations. Public Health Nutr 8: 69-76.

8. Tooze JA, Kipnis V, Buckman DW, Carroll RJ, Freedman LS, et al. (2010) A mixed-effects model approach for estimating the distribution of usual intake of nutrients: the $\mathrm{NCl}$ method. Stat Med 29: 2857-2868.

9. Nusser SM CA, Dodd KW, Fuller WA (1996) A semiparametric transformation approach to estimating usual daily intake distributions. J Am Stat Assoc 91: 1440-1449.

10. Guenther PM, Kott PS, Carriquiry AL (1997) Development of an approach for estimating usual nutrient intake distributions at the population level. J Nutr 127: 1106-1112.

11. Nelson M, Black AE, Morris JA, Cole TJ (1989) Between- and within-subject variation in nutrient intake from infancy to old age: estimating the number of days required to rank dietary intakes with desired precision. Am J Clin Nutr 50: $155-167$

12. Huybrechts I, De Bacquer D, Cox B, Temme EH, Van Oyen $H$, et al. (2008) Variation in energy and nutrient intakes among pre-school children: implications for study design. Eur J Public Health 18: 509-516.

13. Beaton GH, Milner J, Corey P, McGuire V, Cousins M, et al. (1979) Sources of variance in 24-hour dietary recall data: implications for nutrition study design and interpretation. Am J Clin Nutr 32: 2546-2559.

14. Freedman LS, Midthune D, Carroll RJ, Krebs-Smith S, Subar AF, et al.(2004) Adjustments to improve the estimation of usual dietary intake distributions in the population. J Nutr 134: 1836-43.

15. Sawaya AL, Tucker K, Tsay R, Willett W, Saltzman E, et al.(1996) Evaluation of four methods for determining energy intake in young and older women: comparison with doubly labeled water measurements of total energy expenditure. Am J Clin Nutr 63: 491-499.

16. Wu ML, Whittemore AS, Jung DL (1986) Errors in reported dietary intakes. I. Short-term recall. Am J Epidemiol. 124: 826-835.

17. Barrett-Connor E (1991) Nutrition epidemiology: how do we know what they ate? Am J Clin Nutr 54: 182S-187S

18. Murphy SP, Poos MI (2002) Dietary Reference Intakes: summary of applications in dietary assessment. Public Health Nutr 5: 843-849.
19. Asbeck I, Mast M, Bierwag A, Westenhöfer J, Acheson KJ, et al. (2002) Severe underreporting of energy intake in normal weight subjects: use of an appropriate standard and relation to restrained eating. Public Health Nutr 5: 683-690.

20. Heitmann BL (1993) The influence of fatness, weight change, slimming history and other lifestyle variables on diet reporting in Danish men and women aged 35-65 years. Int J Obes Relat Metab Disord 17: 329-336.

21. Goldberg GR, Black AE, Jebb SA, Cole TJ, Murgatroyd PR, et al. (1991) Critical evaluation of energy intake data using fundamental principles of energy physiology: 1. Derivation of cut-off limits to identify under-recording. Eur J Clin Nutr 45: 569-81.

22. FAO/WHO/UNU (1985) Energy and protein requirements. Geneva: World Health Organization.

23. Black AE (2000) The sensitivity and specificity of the Goldberg cut-off for El:BMR for identifying diet reports of poor validity. Eur J Clin Nutr 54: 395-404.

24. Poslusna K, Ruprich J, de Vries JH, Jakubikova M, van't Veer $P$ (2009) Misreporting of energy and micronutrient intake estimated by food records and 24 hour recalls, control and adjustment methods in practice. $\mathrm{Br} \mathrm{J}$ Nutr 101 S73-S85.

25. Liebman M, Cameron BA, Carson DK, Brown DM, Meyer SS (2001) Dietary fat reduction behaviors in college students: relationship to dieting status, gender and key psychosocial variables. Appetite 36: 51-56.

26. Johansson L, Solvoll K, Bjørneboe GE, Drevon CA (1998) Under- and overreporting of energy intake related to weight status and lifestyle in a nationwide sample. Am J Clin Nutr 68: 266-274.

27. Oh SY, Hong MH (1999) Within- and between-person variation of nutrien intakes of older people in Korea. Eur J Clin Nutr 53: 625-629.

28. Nyambose J, Koski KG, Tucker KL (2002) High intra/interindividual variance ratios for energy and nutrient intakes of pregnant women in rural Malawi show that many days are required to estimate usual intake. J Nutr 132: 1313-1318.

29. Jahns L, Carriquiry A, Arab L, Mroz TA, Popkin BM (2004) Within- and betweenperson variation in nutrient intakes of Russian and U.S. children differs by sex and age. J Nutr 134: 3114-3120.

30. Neuhaus JM, Murphy SP, Davis MA. (1991) Age and sex differences in variation of nutrient intakes among U.S. adults. Epidemiology 2: 447-450.

31. Hodson L Skeaff CM, Fielding BA (2008) Fatty acid composition of adipose tissue and blood in humans and its use as a biomarker of dietary intake. Prog Lipid $\operatorname{Re} 47: 348-80$.

32. Mayne ST (2003) Antioxidant nutrients and chronic disease: use of biomarkers of exposure and oxidative stress status in epidemiologic research. J Nutr 133 933S-940S.

33. Black AE, Cole TJ, Wiles SJ, White F (1983) Daily variation in food intake of infants from 2 to 18 months. Hum Nutr Appl Nutr 37: 448-458.

34. Mennen LI, Bertrais S, Galan P, Arnault N, Potier de Couray G, et al. (2002) The use of computerised $24 \mathrm{~h}$ dietary recalls in the French SU.VI.MAX Study: number of recalls required. Eur J Clin Nutr 56: 659-65. 